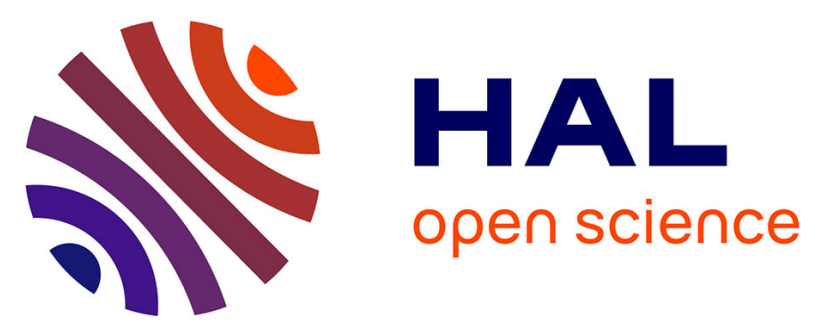

\title{
Parallelized Monte-Carlo using graphics processing units to model cylindrical diffusers used in photodynamic therapy: From implementation to validation
}

\author{
Clément Dupont, Grégory Baert, Serge Mordon, Maximilien Vermandel
}

\section{To cite this version:}

Clément Dupont, Grégory Baert, Serge Mordon, Maximilien Vermandel. Parallelized MonteCarlo using graphics processing units to model cylindrical diffusers used in photodynamic therapy: From implementation to validation. Photodiagnosis and Photodynamic Therapy, 2019, 10.1016/j.pdpdt.2019.04.020 . hal-02524687

\section{HAL Id: hal-02524687 \\ https://hal.science/hal-02524687}

Submitted on 30 Mar 2020

HAL is a multi-disciplinary open access archive for the deposit and dissemination of scientific research documents, whether they are published or not. The documents may come from teaching and research institutions in France or abroad, or from public or private research centers.
L'archive ouverte pluridisciplinaire HAL, est destinée au dépôt et à la diffusion de documents scientifiques de niveau recherche, publiés ou non, émanant des établissements d'enseignement et de recherche français ou étrangers, des laboratoires publics ou privés. 


\title{
Parallelized Monte-Carlo using graphics processing units to model cylindrical diffusers used in photodynamic therapy: From implementation to validation
}

\author{
Clément DUPONT, Grégory BAERT, Serge MORDON, Maximilien VERMANDEL \\ Univ. Lille, Inserm, CHU Lille, U1189 - ONCO-THAI - Image Assisted Laser Therapy for \\ Oncology, F-59000 Lille, France
}

\section{Correspondence:}

Dr. Maximilien VERMANDEL

maximilien.vermandel@univ-lille.fr

\begin{abstract}
:
The Monte-Carlo method is the standard method for computing the dosimetry of both ionizing and non-ionizing radiation. Because this technique is highly time-consuming in conventional implementations, several improvements have recently been developed to speed-up simulations. Among the improvements, the use of graphics processing units (GPU) to parallelize algorithms provides a cost-efficient solution to accelerate the Monte-Carlo method. Parallel implementation of Monte-Carlo using GPU technology is described in the context of photodynamic therapy (PDT) dosimetry. This algorithm has been optimized to compute light emitted from optical fibers with cylindrical diffusers that are used in interstitial PDT applications. A comparison of the experimental measurements used to assess the results of the Monte-Carlo method is detailed. Illumination profiles of several commercially available diffusers are measured using an optical phantom that mimics the optical properties of the brain. Additionally, this Monte-Carlo method is compared to ex-vivo measurements made by a device dedicated to intraoperative PDT treatment of brain tumors.

The results of the GPU Monte-Carlo validation are in accordance with the recommendations of the American Association of Physicists in Medicine. The acceleration obtained with the GPU implementation is in accordance with the literature and is sufficiently fast to be integrated in a treatment planning system dedicated to planning routine clinical interstitial PDT treatments.
\end{abstract}

Keywords: Monte-Carlo modeling, graphics processing unit algorithm, dosimetry, photodynamic therapy, cylindrical diffuser

\section{Introduction}

Photodynamic therapy (PDT) is a non-thermal, energy-based therapy that relies on light exposure after the accumulation of photosensitizers (PS) in tumor cells. The effects of PDT result from the synergy of three elements: a PS, the presence of oxygen and the energy delivered through light exposure at a specific wavelength to excite the PS. The combination of these three components leads to the formation of reactive oxygen species (ROS), including singlet oxygen, which are cytotoxic molecules that damage PS-targeted cells. 
When using a PS with limited selectivity, light only needs to be applied on the areas to be treated (e.g., hematoporphyrin, Photofrin ${ }^{\circledR}[1,2]$ ), and the organs at risk must be avoided. When using a PS with high selectivity (e.g., temoporfin, Foscan ${ }^{\circledR}$ or protoporphyrin IX (PpIX) [3-6]), the margins that are required around the targeted tumor sites can be considerably relaxed.

Several lighting modalities are available and can vary according to the topology of the target zone. Among them, optical fibers with cylindrical diffusers enable the illumination of a large tissue volume. These optical fibers produce an ellipsoidal illumination around the extremity of the diffusing tip. Currently, this type of diffuser is primarily used in clinical applications (interstitial or cavity treatments) because it maximizes the irradiated volume during the procedure and thus, minimizes both the number of fibers that need to be inserted and the treatment time [7].

Different surgical procedures can be considered according to the accessibility of the treatment target. When tumor tissues are easily reachable, such as on the skin or during a surgical procedure, dedicated lighting devices are preferred to better control the illumination. Otherwise, interstitial procedures are used by inserting cylindrical diffusers into the target tumor. Positioning optical fibers usually requires prior simulations to optimize the location of each fiber. Currently, these simulations can be performed with the assistance of a treatment planning system (TPS). Due to recent technological improvements (imaging and computing performance), a few TPSs have been developed for interstitial PDT and for different applications, but these systems remain a work in progress [8-12]. Among the planning steps, computing the impact of PDT on tissues is still a major challenge. PDT dosimetry is a dynamic, complex process that includes several time-dependent features that differ from one individual to another. PDT dosimetry requires the accurate computation of the light dose deposited in biological tissues. Among all models that enable the computation of light propagation, a Monte-Carlo model that simulates the interactions of photons in biological media is the prime candidate for use in PDT dosimetry [13]. However, this model is highly time-consuming when using conventional algorithm implementations. Thus, routine clinical use of the Monte-Carlo method is still limited because of the long simulation time. The improvement of the computing capacity and new technologies have led to recent techniques that accelerate Monte-Carlo simulations [14-17]. Among these improvements, the use of graphics processing units (GPUs) considerably speed up simulations at a limited cost.

In this study, a Monte-Carlo algorithm for light modeling and accelerated using GPU parallelization is described. The purpose of this study is to provide a dosimetric algorithm that is optimized to compute light propagation emitted from interstitial cylindrical diffusers with a computing time that is acceptable for clinical use. The results obtained from Monte-Carlo simulations were then compared with experimental measurements. A homogeneous optical phantom was created to mimic the optical properties of biological tissues. The illumination profiles of five different cylindrical diffusers were measured and compared to the MonteCarlo results. The performance of the algorithm was optimized to a specific GPU card to obtain fast computations. Moreover, this Monte-Carlo algorithm was used to confirm experimental measurements by assessing the dosimetry of a lighting device used in brain surgery. This analysis enables the evaluation of the relevance of our Monte-Carlo model for realistic biological tissues. 


\section{II.Material and methods}

\section{GPU Monte-Carlo implementation}

\section{a. Architecture}

The Monte-Carlo method presented in this study was designed to be implemented in a future TPS dedicated to interstitial photodynamic therapy, with light delivered through cylindrical diffuser tips, for brain cancer treatment [18]. Monte-Carlo simulations rely on modeling light sources according to their location and shape in the patient's brain. This brain is modeled by a 3D matrix of voxels that are classified according to the type of biological medium to which they belong (white matter, grey matter, tumor, edema, necrosis) [19]. These data can be obtained from magnetic resonance imaging (MRI).

The particularity of the GPU programming lies in the architecture of the device used. The term "Host" refers to the CPU and its memory, and the term "Device" refers to the GPU. The parallelized code is equally distributed into several units called "kernels" similar to tiny processing units, that are located in the device. Kernels are grouped into several "blocks", and each kernel instance is called a "thread."

Structure proposed by Fang et al. [15] and the mcxyz code by Jacques et al. inspired our GPU Monte-Carlo method [20, 21].

The input features of the algorithm are defined as:

- $\quad N x, N y$, and Nzare the numbers of voxels in the X-, Y-, and Z-axes, respectively, of the 3D matrix (i.e., the MRI volume).

- $\quad d x, d y$, and $d z$ are the voxel sizes in the $\mathrm{X}-, \mathrm{Y}-$, and Z-axes, respectively.

- $\left(x_{i n}, y_{i n}, z_{i n}\right)$ are the triplet of coordinates corresponding to the beginning of a cylindrical diffuser tip (proximal).

- $\left(x_{\text {out }}, y_{\text {out }}, z_{\text {out }}\right)$ are the triplet of coordinates corresponding to the end of a cylindrical diffuser tip (distal).

Each thread generated on the GPU runs an entire photon history (from photon birth with the initial properties to photon death). Thus, depending on the hardware capacity, several photon histories are simultaneously and independently launched on multiple threads.

The pipeline of the GPU Monte-Carlo simulation and the propagation process of photons is similar to those described by Fang et al. [15], namely:

- A photon is first created at a random position between the two extremities, $\left(x_{i n}, y_{i n}, z_{i n}\right)$ and $\left(x_{\text {out }}, y_{\text {out }}, z_{\text {out }}\right)$ of the emitting part of the cylindrical diffuser that is inserted into the body of the patient. The photon is isotropically orientated and has a weight of 1 .

- A given distance, called the step size, is deduced from the absorption and scattering coefficients. Step size denotes the displacement of the photon through matter at each iteration of the algorithm.

- If the photon does not cross the boundaries of the voxel, it moves to its next location ("Hop" process) and loses weight according to the length of the step size and the absorption property of the voxel ("Drop" process).

- If the photon crosses boundaries of the voxel, it moves at this boundary, loses weight according to the length of its distance to the voxel's boundary and the 
absorption property of the voxel. The remaining step size is computed to continue its propagation.

- A new random direction is computed using optical properties (“Spin" process).

- The steps above are repeated (from the generation of the step size) until the photon's weight can be considered null ("Roulette" process) or the photon exits the entire volume.

Depending on hardware capacities, the required number of simulated photons is typically larger than the number of kernels available. Thus, once a kernel completes a photon history, another photon propagation thread is repeated in the same kernel. Each kernel repeats the entire simulation process until the expected number of photons is reached.

The output of the simulation is a $N x$ by $N y$ by $N z$ absorption matrix (photon_weight/bin), which is normalized to obtain the transport matrix $\left(M_{\text {transport }}[N x, N y, N z], \mathrm{cm}^{-2}\right)$ and multiplied with the power source $\left(\left(P_{\text {source }}, \mathrm{W}\right)\right.$ to obtain the fluence rate $\left(F[N x, N y, N z], \mathrm{W} \cdot \mathrm{cm}^{-2}\right)$.

b. Comparison between CPU and GPU implementations

A comparison between CPU and GPU implementations of the Monte-Carlo algorithm was performed. The same algorithm was distributed on both an Intel Xeon CPU (E5-1603 v3 2.80GHz) and an NVIDIA GPU Quadro K620 (CUDA 7.5 platform).

The only difference of these two implementations was in the pseudorandom number generator (PRNG) used in each technology. The CPU implementation included the native PRNG provided by the standard library stdlib, which is based on a linear congruential generator, whereas the GPU used the tiny Mersenne Twister PRNG, as previously described.

To compare these two implementations, 6 cylindrical diffusers with different lengths of the fiber tip were modeled: 10, 15, 20, 30, 40 and $50 \mathrm{~mm}$, which correspond to the standard lengths provided in the market for PDT applications. The same experimental conditions were implemented in each simulation. All of the cylindrical diffusers were placed in the center of a 40x40x80 mm homogeneous medium with the following optical properties:

- Absorption coefficient: $\mu_{\mathrm{a}}=0.1 \mathrm{~cm}^{-1}$

- Scattering coefficient: $\mu_{\mathrm{s}}=100 \mathrm{~cm}^{-1}$

- Anisotropy coefficient: $g=0.8$

Photons $\left(10^{6}\right)$ were generated in each simulation. The deviation of the fluence rate values were computed in each voxel of the horizontal cut plane of the 40x40x80 mm homogeneous medium. The mean value of each deviation was then computed for each cylindrical diffuser:

$$
\text { mean deviation }=\frac{1}{40 * 80} \sum_{x=0}^{40} \sum_{y=0}^{80}\left|\phi_{\mathrm{CPU}}(x, y)-\phi_{\mathrm{GPU}}(x, y)\right|
$$

c. Statistical noise

The accuracy of the Monte-Carlo simulations improved with the increase of the number of modeled photons. Due to the randomness of this algorithm, the statistical noise was evaluated. Six cylindrical diffusers with different lengths of the fiber tip were modeled: $10,15,20,30$, 40 and $50 \mathrm{~mm}$. The same experimental conditions were implemented in each simulation. All 
of the cylindrical diffusers were placed in the center of a 40x40x80 mm homogeneous medium with the following optical properties:

- Absorption coefficient: $\mu_{\mathrm{a}}=0.1 \mathrm{~cm}^{-1}$

- Scattering coefficient: $\mu_{\mathrm{s}}=100 \mathrm{~cm}^{-1}$

- Anisotropy coefficient: $\mathrm{g}=0.8$

Photons $\left(10^{6}\right)$ were generated in each simulation. Simulation were performed 10 times for each cylindrical diffuser; 60 Monte-Carlo simulations were achieved.

The standard deviations of these 10 realizations were computed in each voxel of the horizontal cut plane of the 40x40x80 mm homogeneous medium, i.e., 3200 standard deviations were computed for each cylindrical diffusor simulation.

\section{d. Cylindrical diffuser modeling}

In most cases, cylindrical diffusers are preferred over flat-cleaved fibers for interstitial PDT applications because they allow a shorter treatment duration [7]. Several Monte-Carlo methods have already been described in the literature to model cylindrical diffusers [22-26]. Comprehensive modeling of the cylindrical tip has been detailed by Baran et al. [27] by describing four of the tip's components: the core and cladding of the fiber, a diffusive medium and a dielectric reflector. This model produces a more realistic simulation of the light distribution by the diffuser tip. This model provides a better prediction of the fluorescence detection in surrounding tissues and a similar fluence rate distribution compared to a simple linear array of point sources as proposed in previous works [25, 28].Thus, regarding this literature, the linear point source model has been chosen to predict fluence rate distribution around cylindrical diffusers.

Our Monte-Carlo method describes the cylindrical diffuser tip as an isotropic line source. The location of photon creation was uniformly randomized between the two triplets of coordinates $\left(\mathrm{x}_{\text {in }}, \mathrm{y}_{\text {in }}, \mathrm{z}_{\text {in }}\right.$ and $\mathrm{x}_{\text {out }}, \mathrm{y}_{\text {out }}, \mathrm{z}_{\text {out }}$ ) corresponding to the proximal and distal ends of the cylindrical diffuser tip. Each photon was then isotropically launched into the surrounding tissues. Modeling all of the different parts of the fiber tip was considered unnecessary because a voxel size equivalent to the standard MRI resolution (i.e., one millimeter) was used and the diameter of the fiber tip was smaller than one millimeter.

Nevertheless, our Monte-Carlo model takes the light distribution profile of the cylindrical diffusers used in PDT into account. To implement this property, the light distribution profiles of five different cylindrical diffusers were measured and normalized based on both the light power and the length of the fiber tip (figure 1). 


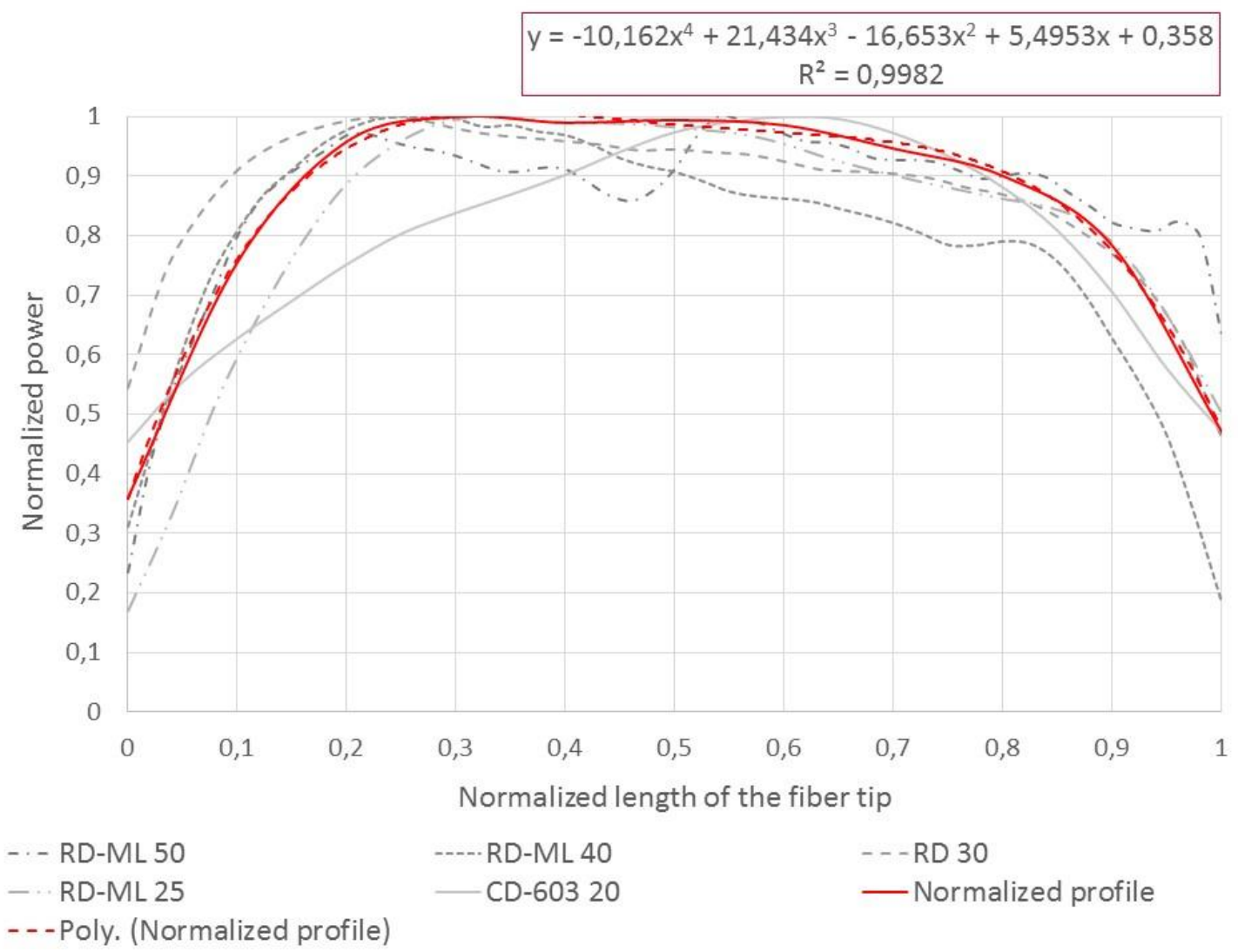

Figure 1: In grey, the normalized light emission profiles of five cylindrical diffusers (Medlight, Ecublens, Switzerland (RD and RD-ML models) and Biolitec GmbH, Jena, Germany (CD model)) are shown. In red, the mean normalized profile used in the Monte-Carlo model to weight photons at their creation are shown.

Because these emission profiles were only measured in a single diffuser for each type of diffuser, a mean normalized profile was computed, and a $4^{\text {th }}$ order polynomial was used to estimate this mean normalized profile (equation 2). This empirical equation was then applied to all of the photons created in our Monte-Carlo model to adjust their initial weight $w_{\text {photon }}$ according to the location of their creation along the diffuser tip, in order to tend toward a better approximation of the fluence rate emitted in tissues.

$$
\begin{gathered}
w_{\text {photon }}=-10.162 x^{4}+21.434 x^{3}-16.653 x^{2}+5.4953+0.358 \\
\text { with } x=\sqrt{\left(\left|x_{\text {distal }}-x\right|\right)^{2}+\left(\left|y_{\text {distal }}-y\right|\right)^{2}+\left(\left|z_{\text {distal }}-z\right|\right)^{2}} \\
\text { e. Performance }
\end{gathered}
$$

Several configurations were tested to optimize the number of blocks and the threads used per block to obtain the fastest execution of the Monte-Carlo simulation. To compare the performance of the Monte-Carlo simulation with a reference framework, a homogeneous medium was implemented with the following optical properties:

- Absorption coefficient: $\mu_{\mathrm{a}}=0.1 \mathrm{~cm}^{-1}$

- Scattering coefficient: $\mu_{\mathrm{s}}=100 \mathrm{~cm}^{-1}$

- Anisotropy coefficient: $g=0.8$ 
Photons $\left(10^{5}\right)$ were generated to simulate the light distribution from a $40 \mathrm{~mm}$ cylindrical diffuser placed at the center of a 40x40x80 mm homogeneous slab of the previously described medium. By varying the distribution of threads used by the GPU, the mean computing time was reduced by empirically looking for the fastest configuration.

Among the metrics available for measuring the performance of a parallelized code, Amdhal's law $[29,30]$ defines the acceleration $A_{n p}$ due to parallelism as:

$$
A_{n p}=\frac{1}{(1-\alpha)+\frac{\alpha}{n p}}
$$

where $\alpha$ is the proportion of execution time that benefits from parallelized implementation and $n p$ is the number of kernels.

The parallelization efficiency $E_{n p}$ is the ratio of the acceleration $A_{n p}$ and the number of kernels $n p$ :

$$
E_{n p}=\frac{A_{n p}}{n p}=\frac{1}{(1-\alpha) \cdot n p+\alpha}
$$

\section{Monte-Carlo validation by comparison with experimental measurements}

a. Phantom design

For the purpose of validating this GPU Monte-Carlo implementation, a phantom of known optical properties was created to compare the experimental and simulated measurements. The most popular and easiest way to build such a phantom is to mix an Intralipid liquid, India ink and water [31-35]. Such phantoms have been extensively studied, and calculations of the quantities to be mixed rely on the multi-center study of Spinelli et al. [34], published in 2014. In the Spinelli study, the same optical phantom based on Intralipid and India ink was characterized using nine different methods in nine different research laboratories from six countries. They proposed a mathematical model for the masses of India ink and Intralipid that needed to be mixed to obtain the desired optical properties. The values of absorption $\mu_{\mathrm{a}}=0.2$ $\mathrm{cm}^{-1}$ and reduced scattering $\mu_{\mathrm{s}}^{\prime}=20 \mathrm{~cm}^{-1}$ correspond to the mean optical properties of human brain tissue [26, 36-38]. These values will be considered references in this study.

\section{b. Calibration and experimental setup}

An experiment was designed to characterize the fluence rate $\left(\mathrm{W} \cdot \mathrm{cm}^{-2}\right)$ of different diffusers that are commonly used in PDT treatment within the phantom. The only variable that can be measured in a light dosimetry experiment is the light power $(\mathrm{W})$. To convert this power value $P_{\text {measured }}(\mathrm{W})$ into a fluence rate $\phi_{\text {measured }}\left(\mathrm{W} \cdot \mathrm{cm}^{-2}\right)$, a calibration factor $C F_{\text {medium }}$ that depends on the probe and the surrounding medium was used:

$$
\phi_{\text {measured }}=\mathrm{CF}_{\text {medium }} \cdot \mathrm{P}_{\text {measured }}
$$

In their two papers, Marijnissen and Star provide a complete methodology to obtain this calibration factor $C F_{\text {medium }}[39,40]$, which is also recommended by the American Association of Physicists in Medicine (AAPM) [41, 42]. This factor is the combination of a calibration factor determined in air $C F_{\text {air }}$ and four other corrective coefficients. This methodology has also been described in a previous study [43]. 
A cylindrical diffuser was inserted in a $15 \times 15 \times 15 \mathrm{~cm}$ black box to avoid light reflection and fixed so that it was as straight as possible. An isotropic sensor (IP85, Medlight, Ecublens, Switzerland) was fixed to a mobile support and located at precise coordinates, e.g., against the cylindrical diffuser. Once the sensor and the optical fiber were in place, the phantom was poured inside the black box to completely fill it. A Ceralas 635 (Biolitec, Jena, Germany) laser source was then connected to the SMA connector of the fiber. Power measurements were obtained along the horizontal cut plane of the cylindrical diffuser. The sensor was moved with a step size of one millimeter $(\Delta x, \Delta y)$ from the beginning to the end of the cylindrical diffuser ( $y$-axis) until it reached a $1 \mathrm{~cm}$ distance from light source ( $x$-axis) (see figure 2).

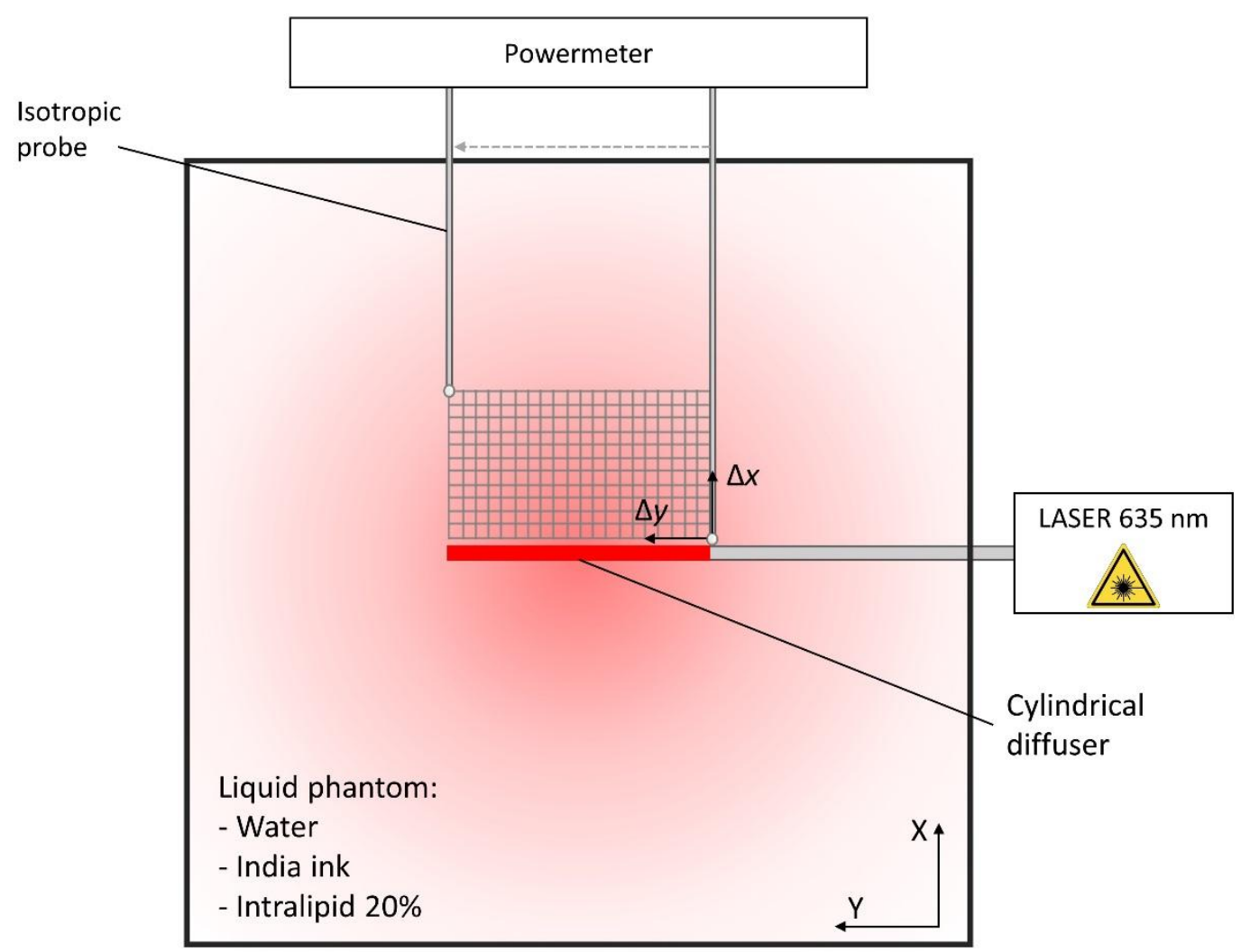

Figure 2: Grid of the power measurement positions.

A power of $500 \mathrm{~mW}$ was emitted from the laser source. Once the power was measured, the value was multiplied by the calibration factor to obtain a fluence rate.

These fluence rates were then compared to those obtained by Monte-Carlo modeling. In each simulation, $10^{7}$ photons were modeled according to the previously described rules. The tip of the modeled cylindrical diffuser was placed at the center of a 40x40x80 homogeneous slab. The same optical properties (absorption, scattering and anisotropy) were applied to compare the experimental results with the Monte-Carlo results. 
To compare the results from both the reference experimental fluence rate measurements $\phi_{\text {measured }}$ and the Monte-Carlo modeling $\phi_{\mathrm{MC}}$, the relative deviation at different locations from the fiber tip and the mean relative deviation were defined as:

$$
\begin{gathered}
\text { Relative deviation }_{x}(x)=\frac{\Delta y}{L_{\text {tip }}} \sum_{y=0}^{L_{\text {tip }}} \frac{\left|\phi_{\text {measured }}(x, y)-\phi_{\mathrm{MC}}(x, y)\right|}{\phi_{\text {measured }}(x, y)} \\
\text { Mean relative deviation } n_{x}=\frac{1}{8} \sum_{x=0}^{8} \text { Relative deviation }(x)^{8}
\end{gathered}
$$

and

$$
\begin{aligned}
& \text { Relative deviation }_{y}(y)=\frac{1}{8} \sum_{x=0}^{8} \frac{\left|\phi_{\text {measured }}(x, y)-\phi_{\mathrm{MC}}(x, y)\right|}{\phi_{\text {measured }}(x, y)} \\
& \text { Mean relative deviation } n_{y}=\frac{\Delta y}{L_{\text {tip }}} \sum_{y=0}^{L_{\text {tip }}} \text { Relative deviation }(y)^{\text {and }}
\end{aligned}
$$

where $L_{\text {tip }}$ is the length of the fiber tip ( $\mathrm{mm}$ ),

$\phi_{\text {measured }}(x, y)$ is the measured fluence rate (equation 5$)\left(\mathrm{W} \cdot \mathrm{cm}^{-2}\right)$,

$\phi_{\mathrm{MC}}(x, y)$ is the fluence rate obtained with the Monte-Carlo algorithm $\left(\mathrm{W} \cdot \mathrm{cm}^{-2}\right)$, and $\Delta y$ is the step size of the $y$-axis $(\mathrm{mm})$

Values were measured every $1 \mathrm{~mm}$ from the light source $(x=0)$ to a distance of approximately $x=8 \mathrm{~mm}$. After reaching this distance, the power measurements were under the microwatt scale.

Finally, five different cylindrical diffusers were used for measurements and modeled with Monte-Carlo simulation.

The light transmission coefficient for each cylindrical diffuser was measured using three different lasers equipped with an integrating sphere: Ceralas 635 (Biolitec, Jena, Germany), DIOMED 630 PDT (DIOMED, Cambridge, United Kingdom) and ML7710-630-6K (Modulight, Tampere, Finland). This coefficient defines the ratio of the light power emitted into the fiber and the output light power obtained around the cylindrical diffuser tip. The light transmission of each fiber was measured with the three laser sources. The mean of these measurements $\left(T_{\text {transmission }}, \%\right)$ was used to weight the power source factor $\left(P_{\text {source }}, \mathrm{W}\right)$ that was used in combination with the transport matrix $\left(M_{\text {transport }}[N x, N y, N z], \mathrm{cm}^{-2}\right)$ to obtain the fluence rate $\left(F[N x, N y, N z], \mathrm{W} \cdot \mathrm{cm}^{-2}\right)$ matrix of the Monte-Carlo model (see equation 10).

$$
F[N x, N y, N z]=M_{\text {transport }}[N x, N y, N z] \cdot P_{\text {power }} \cdot T_{\text {transmission }}
$$

Five different cylindrical diffusers were used to compare the Monte-Carlo simulation and the experimental measurements (see table 1).

\begin{tabular}{|c|c|c|c|c|c|}
\hline $\begin{array}{c}\text { Manufacturer and } \\
\text { model of the fiber }\end{array}$ & $\begin{array}{c}\text { Length of } \\
\text { the tip } \\
(\mathbf{m m})\end{array}$ & $\begin{array}{c}\text { Diameter } \\
(\mathbf{m m})\end{array}$ & $\begin{array}{c}\text { Core } \\
\text { diameter } \\
(\boldsymbol{\mu m})\end{array}$ & $\begin{array}{c}\text { Numerical } \\
\text { aperture } \\
(\mathbf{d i m e n s i o n l e s s})\end{array}$ & $\begin{array}{c}\text { Transmission } \\
\text { measured }\end{array}$ \\
\hline Biolitec, CD 603-20 & 20 & 1.65 & 600 & 0.37 & $93 \%$ \\
\hline Medlight, RD-ML 25 & 25 & 1 & 400 & 0.37 & $87 \%$ \\
\hline
\end{tabular}




\begin{tabular}{|c|c|c|c|c|c|}
\hline Medlight, RD 30 & 30 & 0.98 & 500 & 0.48 & $71 \%$ \\
\hline Medlight, RD-ML 40 & 40 & 1 & 400 & 0.37 & $87 \%$ \\
\hline Medlight, RD-ML 50 & 50 & 1 & 400 & 0.37 & $87 \%$ \\
\hline
\end{tabular}

Table 1: Characteristics of the five cylindrical diffusers used in experiments

These five cylindrical diffusers, from two optical fiber manufacturers (Medlight, Ecublens, Switzerland and Biolitec GmbH, Jena, Germany), represent the standard optical fiber dimensions used in interstitial PDT.

\section{Monte-Carlo validation by comparison with ex-vivo measurements}

After assessing the Monte-Carlo algorithm in a homogeneous medium, evaluation in a more realistic environment was necessary. Thus, the Monte-Carlo model proposed here was used to confirm experimental ex-vivo measurements made by a specific device described in a previous study [43, 44].

This device has been designed for intraoperative PDT treatment of brain tumors (see figure 3a) and is already being assessed in a phase I clinical study [45]. This device is composed of a balloon inflated with a diffusing solution and inserted into the patient's brain during surgery. A $70 \mathrm{~mm}$ cylindrical diffuser is placed inside a fiber guide composed of a borosilicate glass tube and a stainless-steel tube.

For different volumes of diffusing solution injected into the device, power measurements have been made in the brain tissue around the balloon to assess the fluence rate emitted and to compute the treatment time. The methodology is described in Dupont et al. [43].

These measurements were then compared to Monte-Carlo modeling to evaluate the relevance of the measurements and to validate the Monte-Carlo model in a heterogeneous environment (ex-vivo calf brain). Ten volumes of the injected diffusing solution were simulated: from 50 $\mathrm{mL}$ to $500 \mathrm{~mL}$ with a step of $50 \mathrm{~mL}$. The balloon was designed with an ellipsoidal shape. The dimensions of each configuration were measured (see table 2):

\begin{tabular}{|c|c|c|c|}
\hline $\begin{array}{c}\text { Volume of the injected diffusing solution } \\
(\mathbf{m L})\end{array}$ & $\begin{array}{c}\text { Width } \\
(\mathbf{c m})\end{array}$ & $\begin{array}{c}\text { Length } \\
(\mathbf{c m})\end{array}$ & $\begin{array}{c}\text { Volume of the ellipsoid } \\
\left.\mathbf{( c m}^{\mathbf{3}}\right)\end{array}$ \\
\hline 50 & 4.1 & 7.2 & 63.37 \\
\hline 100 & 5.1 & 7.5 & 102.14 \\
\hline 150 & 5.9 & 8.0 & 145.81 \\
\hline 200 & 6.4 & 8.7 & 186.59 \\
\hline 250 & 6.9 & 9.1 & 226.85 \\
\hline 300 & 7.2 & 9.6 & 260.58 \\
\hline 350 & 7.8 & 10.5 & 334.49 \\
\hline 400 & 8.5 & 11.1 & 419.91 \\
\hline 450 & 8.7 & 11.5 & 455.76 \\
\hline 500 & 8.9 & 11.8 & 489.4 \\
\hline
\end{tabular}

Table 2: Balloon dimensions used to generate the ellipsoid during Monte-Carlo simulations.

Using these dimensions and their respective optical properties, four different materials that compose the device (see figure $3 b$ ) were modeled: standard brain tissues $\left(\mu_{\mathrm{a}}=0.2 \mathrm{~cm}^{-1}, \mu_{\mathrm{s}}=\right.$ 
$\left.160 \mathrm{~cm}^{-1}\right)$, diffusing solutions $\left(\mu_{\mathrm{a}}=10^{-3} \mathrm{~cm}^{-1}, \mu_{\mathrm{s}}=10 \mathrm{~cm}^{-1}\right)$, stainless steel tubes $\left(\mu_{\mathrm{a}}=10^{5} \mathrm{~cm}^{-}\right.$ $\left.{ }^{1}, \mu_{\mathrm{s}}=1.0 \mathrm{~cm}^{-1}\right)$ and borosilicate glass tubes $\left(\mu_{\mathrm{a}}=10^{-4} \mathrm{~cm}^{-1}, \mu_{\mathrm{s}}=0.3 \mathrm{~cm}^{-1}\right)$. The $70 \mathrm{~mm}$ cylindrical diffuser (RD-ML 70, Medlight, Ecublens, Switzerland) was placed at the center of the borosilicate glass.

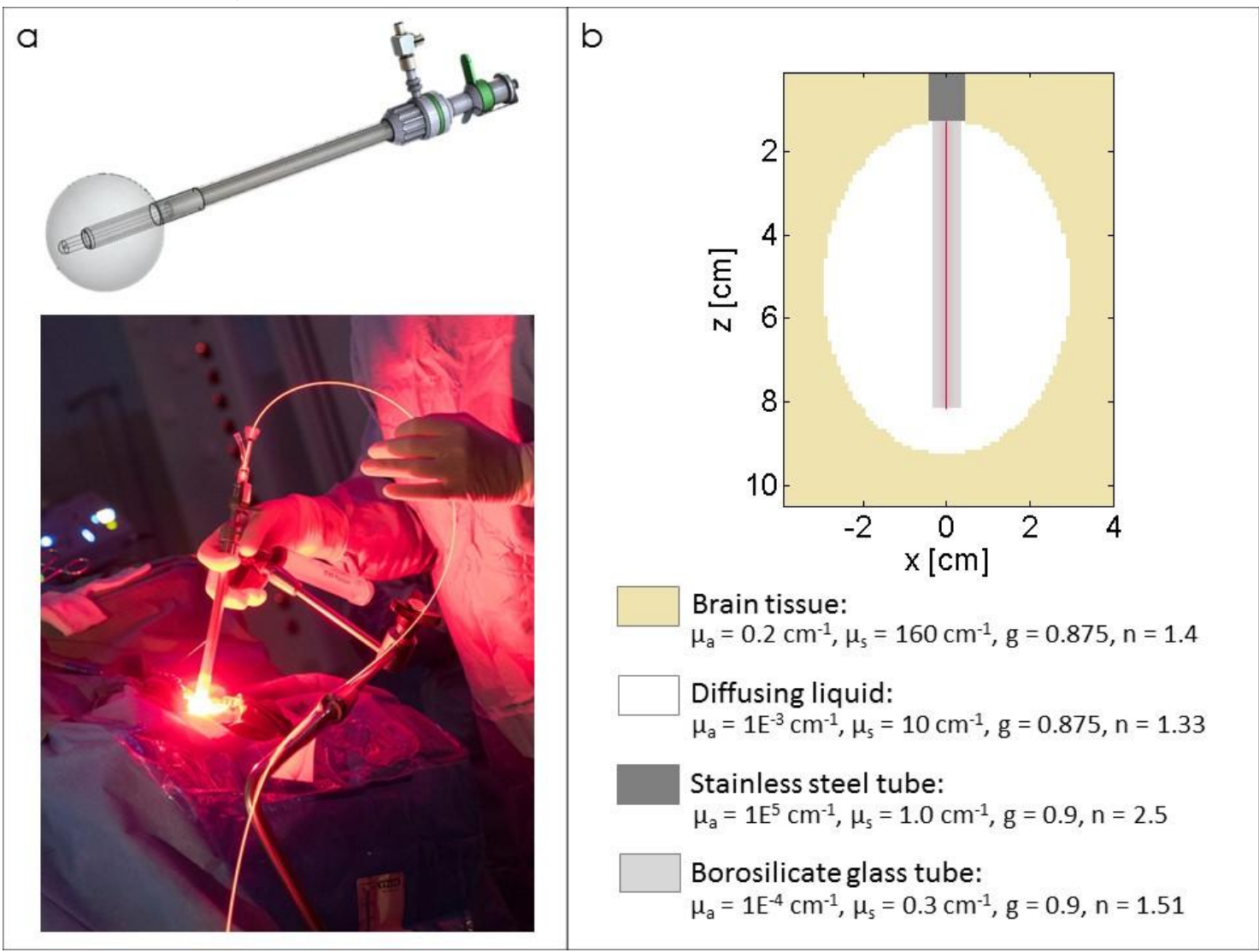

Figure 3: a) Lighting applicator in which the fiber guide is inserted into the balloon to guide the 70 $\mathrm{mm}$ cylindrical diffuser and its clinical application. b) Modeling of the device filled with $150 \mathrm{~mL}$ of a diffusing solution. The $70 \mathrm{~mm}$ long cylindrical diffuser was located at the center of the borosilicate glass tube.

\section{Results}

\section{Monte-Carlo implementation}

\section{a. Comparison between CPU and GPU implementations}

To validate the shift from CPU to GPU technology, 6 different cylindrical diffusers were modeled using the Monte-Carlo algorithm with CPU and GPU implementations. A comparison of the fluence rates led to a mean value of absolute deviation of $3.27 \mathrm{~mW} / \mathrm{cm}^{2}$ (relative deviation of $8.08 \mathrm{~mW} / \mathrm{cm}^{2}$, max value of $10.49 \mathrm{~mW} / \mathrm{cm}^{2}$ ). These values were negligible compared to maximum values of the fluence rate achieved, which were on the order of several watts. Thus, the GPU implementation of the Monte-Carlo algorithm does not interfere with the accuracy of the modeled photons and provides the same results as the CPU implementation. 


\section{b. Statistical noise}

By running 60 Monte-Carlo simulations (10 realizations of 6 different cylindrical diffusers), standard deviations were computed in each voxel of the horizontal cut plane. In each simulation, the minimum computed standard deviation varied between 0 and $6.1 \times 10^{-}$ ${ }^{6} \mathrm{~mW} / \mathrm{cm}^{2}$. These low values can be explained by the absence of photons far from the light source due to the optical properties of the medium. The maximum standard deviation values varied between 6.8 to $13.3 \mathrm{~mW} / \mathrm{cm}^{2}$. This maximum standard deviation is related to a fluence rate of $2.19 \times 10^{3} \mathrm{~mW} / \mathrm{cm}^{2}$. Using a $95 \%$ confidence interval, a margin of error of $26.6 \mathrm{~mW} / \mathrm{cm}^{2}$ is defined (signal to noise ratio of about 82.3). This margin of error can be considered as a statistical noise.

\section{c. Performance}

All the following tests were performed with an Intel Xeon CPU E5-1603 v3 2.80GHz and an NVIDIA GPU Quadro K620. The CUDA 7.5 platform was used to implement our MonteCarlo algorithm.

Several configurations of the number of blocks and the threads used per block were tested to obtain the fastest execution time for the algorithm.

First, the number of threads per block was set to 512 and the number of blocks was varied from 1 to 1024. As shown in the figure $4 \mathrm{a}$, a strong dependence of the number of employed blocks on computing time was observed. The best solution to improve GPU performance was the 32-block configuration.

Then, with the 32-block configuration, the number of threads per block was varied from 8 to 512 (see figure 4b). The fastest configuration for our GPU configuration was 32 blocks with 256 threads per block. With this configuration, 8192 threads, or photon histories, were simultaneously generated. The time required to perform this simulation was approximately $1762 \mathrm{~ms}$. 

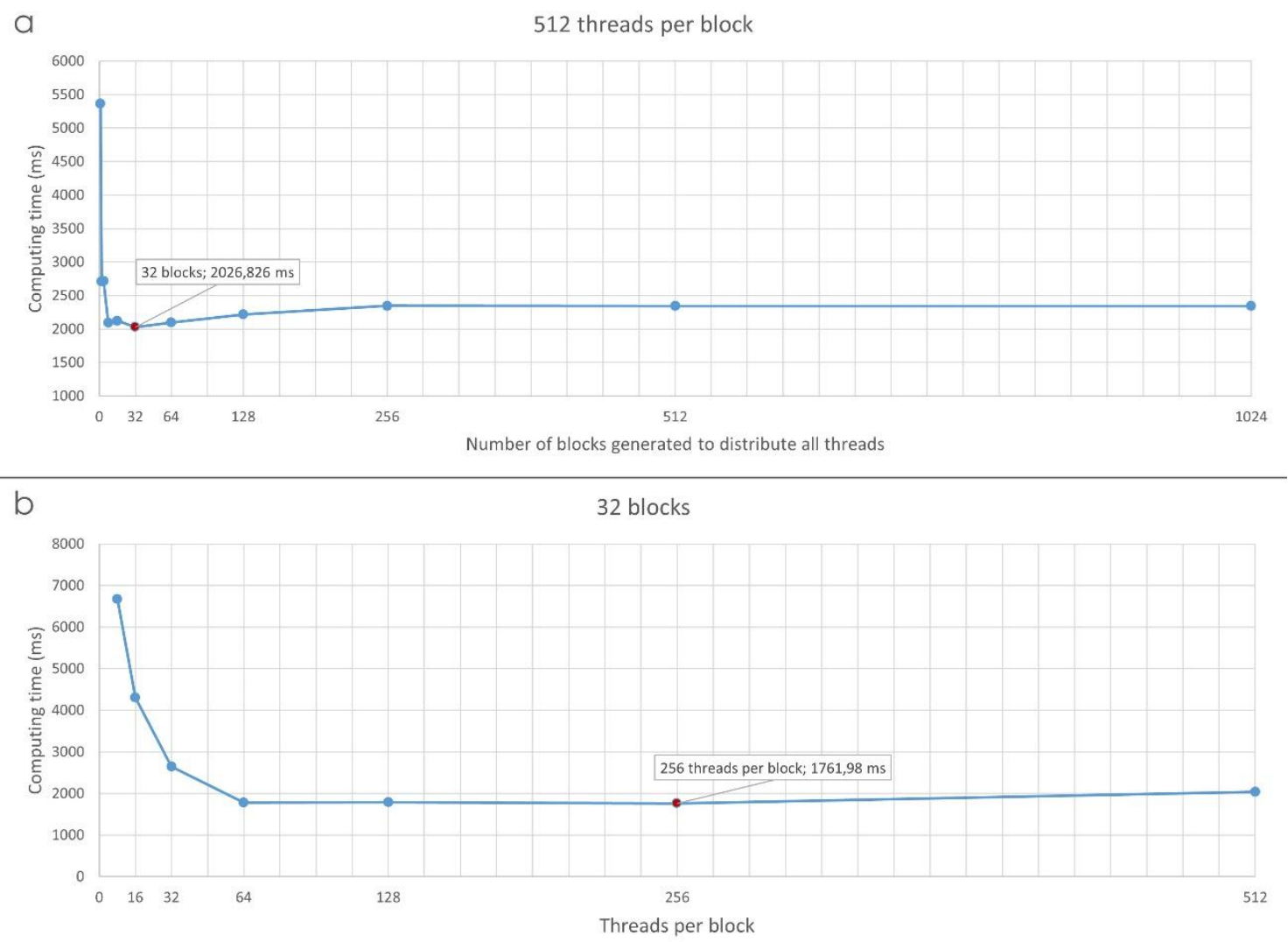

Figure 4: Computing time (ms) (a) as a function of the number of blocks with 512 threads per block and (b) as a function of the number of threads per block with 32 blocks.

The time required to perform the same Monte-Carlo simulation with a CPU configuration was approximately $1341.35 \mathrm{~s}$. Thus, the resulting acceleration and efficiency, previously defined by the Amdhal's law [29, 30], were 761.3 and 0.0929 , respectively. The proportion of execution time $\alpha$ that benefited from parallelized implementation was 0.9988 .

\section{Monte-Carlo validation}

Once the calibration factor was estimated, each power measurement was converted into a fluence rate using equation 5 . The relative deviations in both $\mathrm{x}$ - and $\mathrm{y}$-axes were obtained (equations 6 to 9) for each of the five cylindrical diffusers (see figure 5 and 6). 


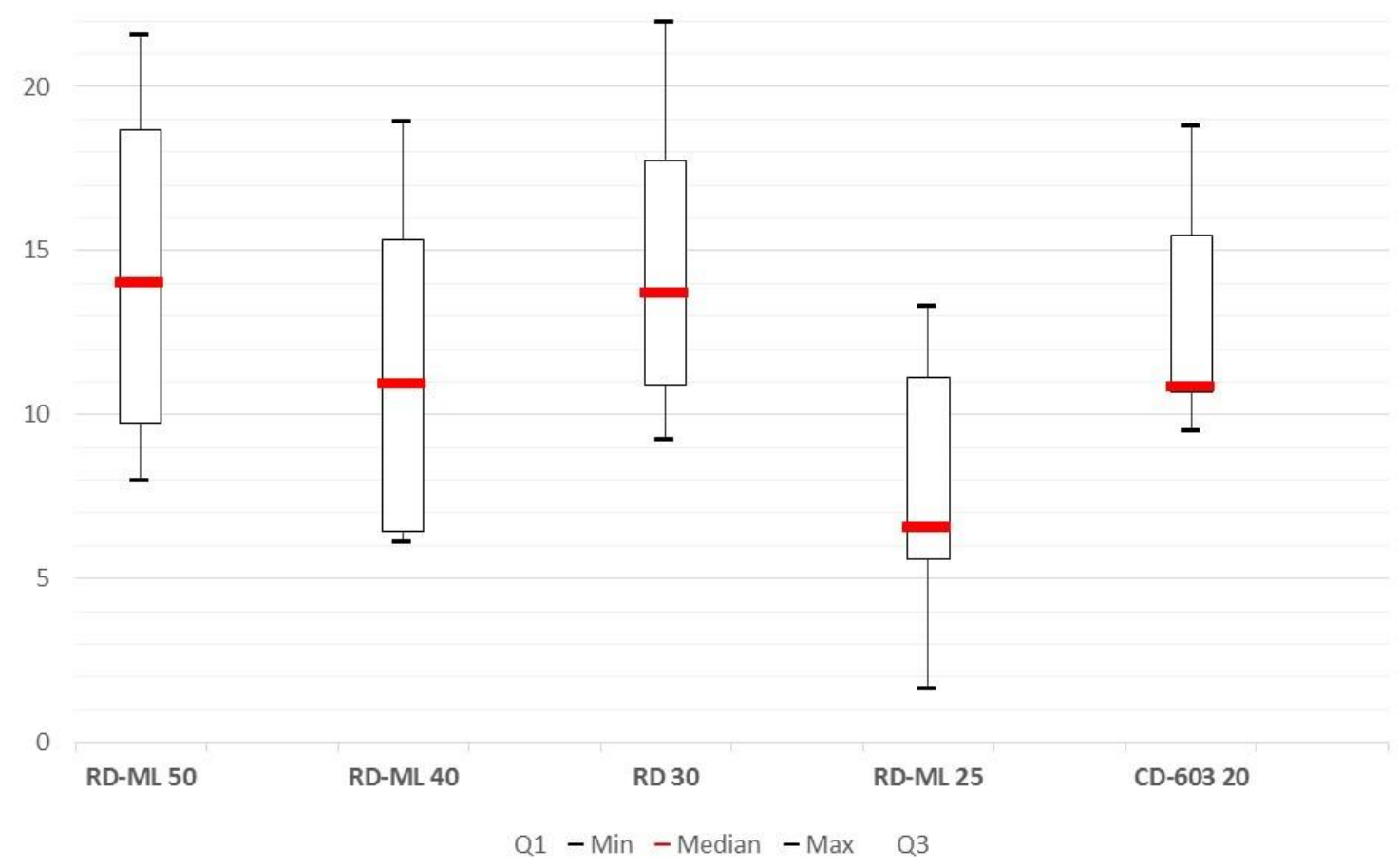

Figure 5: Box plot showing the relative deviations according to the $x$-axis (distances to the fiber tip) (equation 6).

A mean of all the deviations along the $\mathrm{x}$-axis (equation 6) of approximately $12.01 \% \pm 7.62$ was computed (see table 3 ).

\begin{tabular}{|c|c|}
\hline $\begin{array}{c}\text { Manufacturer and model of the } \\
\text { fiber }\end{array}$ & $\begin{array}{c}\text { Mean relative deviations along the x-axis (standard } \\
\text { deviation) - (\%) }\end{array}$ \\
\hline Biolitec, CD 603-20 & $14.55(9.15)$ \\
\hline Medlight, RD-ML 25 & $11.19(4.51)$ \\
\hline Medlight, RD 30 & $14.11(9.78)$ \\
\hline Medlight, RD-ML 40 & $7.54(4.03)$ \\
\hline Medlight, RD-ML 50 & $12.66(10.63)$ \\
\hline Mean & $\mathbf{1 2 . 0 1 ( 7 . 6 2 )}$ \\
\hline
\end{tabular}

Table 3: Mean relative deviations and standard deviation for each cylindrical diffuser (equation 7). 


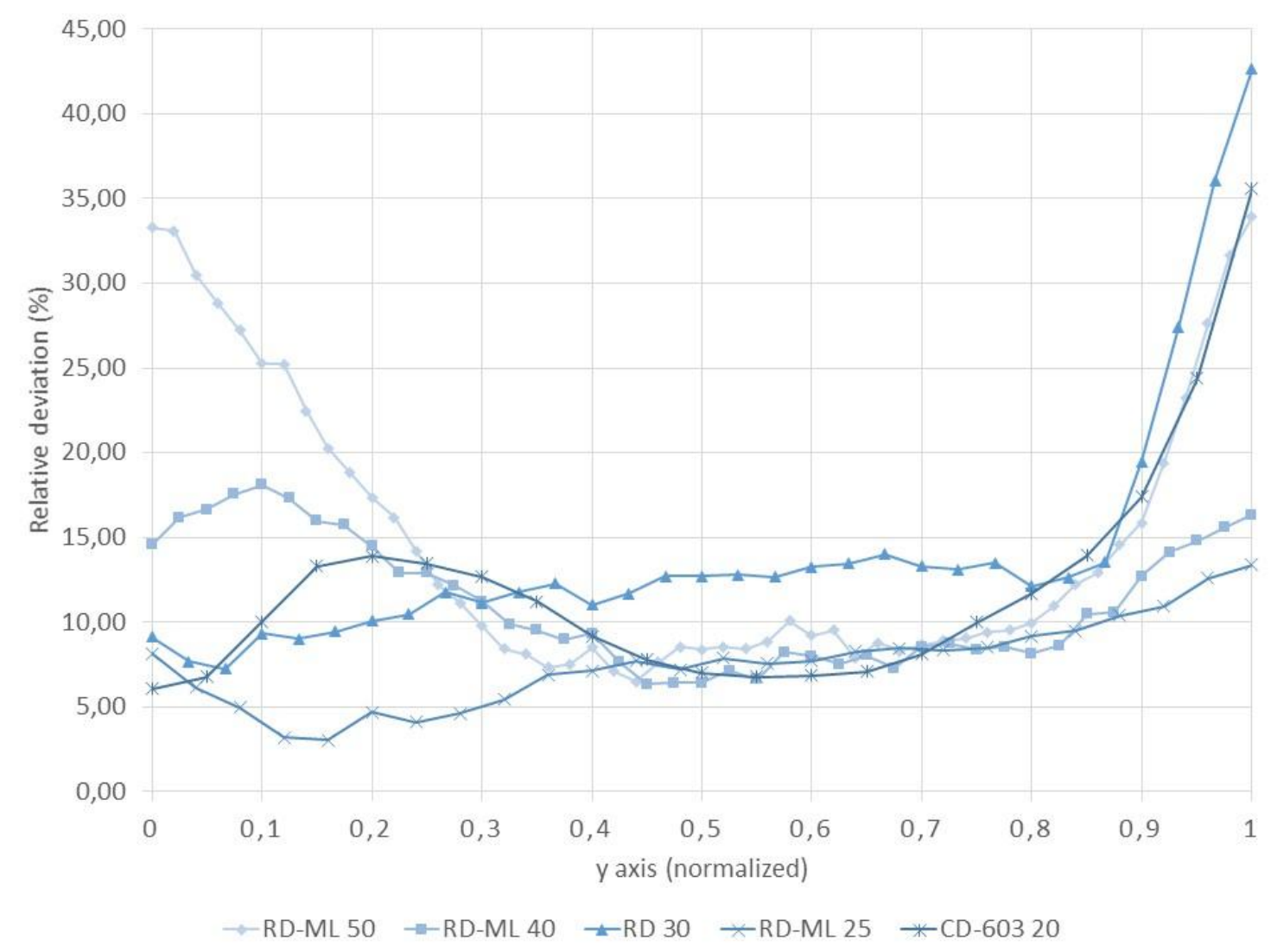

Figure 6: Relative deviation along the $y$-axis (along the fiber tips of each cylindrical diffuser) (equation 8 ). For visibility purposes, the standard deviations are not displayed.

A mean of all deviations along the y-axis (equation 8) of approximately $11.96 \% \pm 6.47$ was computed (see table 4).

\begin{tabular}{|c|c|}
\hline $\begin{array}{c}\text { Manufacturer and model of the } \\
\text { fiber }\end{array}$ & $\begin{array}{c}\text { Mean relative deviations along the y-axis (standard } \\
\text { deviation) }-(\%)\end{array}$ \\
\hline Biolitec, CD 603-20 & $12.06(7.90)$ \\
\hline Medlight, RD-ML 25 & $7.54(5.24)$ \\
\hline Medlight, RD 30 & $14.11(7.41)$ \\
\hline Medlight, RD-ML 40 & $11.19(5.81)$ \\
\hline Medlight, RD-ML 50 & $14.93(5.98)$ \\
\hline Mean & $\mathbf{1 1 . 9 6 ( 6 . 4 7 )}$ \\
\hline
\end{tabular}

Table 4: Mean relative deviations and standard deviation for each cylindrical diffuser (equation 9).

\section{Validation by comparison with ex-vivo measurements}

The relative deviation of the fluence rates against the balloon wall obtained from the power measurements and from the Monte-Carlo method were computed (see table 5).

\begin{tabular}{|l|c|c|}
\hline $\begin{array}{l}\text { Volume of the } \\
\text { balloon }(\mathrm{mL})\end{array}$ & $\begin{array}{c}\text { Fluence rate at the balloon border (standard } \\
\text { deviation) }-\left(\mathrm{mW} \cdot \mathrm{cm}^{-2}\right)\end{array}$ & Relative deviation (\%) \\
\hline
\end{tabular}




\begin{tabular}{|c|c|c|c|}
\hline & $\begin{array}{c}\text { Monte-Carlo } \\
\text { simulation }\end{array}$ & Ex vivo experiments & \\
\hline 50 & $280.82(18.47)$ & $305.79(72.86)$ & 8.89 \\
\hline 100 & $192.93(4.86)$ & $215.94(27.98)$ & 11.93 \\
\hline 150 & $175.45(21.13)$ & $179.52(7.02)$ & 2.13 \\
\hline 200 & $139.06(6.54)$ & $115.54(7.42)$ & 16.91 \\
\hline 250 & $118.30(6.42)$ & $101.36(14.74)$ & 14.44 \\
\hline 300 & $98.23(20.56)$ & $84.65(15.15)$ & 13.82 \\
\hline 350 & $86.06(14.32)$ & $75.56(6.76)$ & 12.19 \\
\hline 400 & $66.28(14.82)$ & $70.50(11.66)$ & 6.38 \\
\hline 450 & $59.53(22.37)$ & $57.19(5.33)$ & 3.93 \\
\hline 500 & $53.10(11.13)$ & $52.02(11.63)$ & 2.03 \\
\hline & & Mean & $9.29 \%$ \\
\hline
\end{tabular}

Table 5: Comparison of the fluence rates obtained from the Monte-Carlo simulations and ex vivo measurements.

\section{Discussion}

Several different approaches enable the propagation of light to be computed. Among them, Monte-Carlo remains a standard method. Despite its high accuracy, this method is rarely used because of the long computational time required to perform a simulation. With recent highcapacity computing and technologies that enable parallel computing, the Monte-Carlo method can now reach an acceptable computing time for routine clinical use.

This study introduces a code fully dedicated to GPU modeling of the cylindrical diffusers used in PDT. To evaluate the output of the algorithm, i.e., the fluence rate emitted from light sources, a phantom was created to mimic particular optical properties. The experimental results were compared to Monte-Carlo simulations, and the mean relative deviations validated the accuracy of the Monte-Carlo model: $12.01 \%$ in the $x$-axis (variation along the tissue depth) and $11.96 \%$ in the $y$-axis (variation along the light emission profile of the cylindrical diffuser). Although these values may appear high, several biases can explain these relative deviations.

The precision of the weight of every compound used to create the optical phantom is critical. Particularly, the mass of India ink injected into the phantom has a strong impact on the absorption coefficient. A slight measurement deviation during the weighing of the India ink can provoke a shift in the phantom absorption. Therefore, the optical coefficients used to simulate the phantom in the Monte-Carlo model are based on the assumption that the phantom is composed of these same coefficients.

The precision of the location of the isotropic probe in the phantom with respect to the cylindrical diffuser position was manually evaluated. Once the liquid optical phantom was poured inside the black box, the isotropic probe could not be seen. It was then impossible to check the position of the bulb during the experiment.

The inhomogeneity of the illumination profiles of the cylindrical diffusers was measured on each diffuser tip and showed a strong variation of illumination profiles. Additionally, the light 
transmission of each cylindrical diffuser was measured with three different diode laser systems with an integrating sphere. Ideally, the heterogeneity and transmission of every cylindrical diffuser should be measured and considered when planning therapy to achieve an accurate dosimetry. Nonetheless, we decided to insert a mean light-emission profile in our Monte-Carlo model to obtain a more realistic linear distribution of photons and thus to model the heterogeneity of the cylindrical diffuser used in PDT. This refinement slightly improved the estimation of fluence rate around the diffuser tip.

The American Association of Physicists in Medicine advised that due to the potential variations caused by many factors, the error in PDT light dosimetry can easily exceed $10 \%$ to $20 \%$ [41, 42]. Considering the biases and measurement uncertainties encountered in this study, the proposed Monte-Carlo model can be accepted.

In the clinical context, the strong optical heterogeneity of brain tissues, particularly in the GBM, induces multiple perturbations for light propagation. Additionally, the optical coefficients provided by different studies $[46,47]$ of the different structures encountered in patient brains show strong variations. It appears to be difficult to model real light propagation through heterogeneous structures using the optical coefficients measured from other brains. Nevertheless, the Monte-Carlo simulation remains the best alternative to reach an estimation of the light distribution.

Regarding the speed gains of the GPU implementation, performance optimization leads to an acceleration of a factor of approximately 760 compared to the CPU version. One of the main drawbacks of the use of GPU technology is that each computing code is optimized to run on a specific GPU device. Thus, the optimization process of the GPU computing program is highly dependent on the type of GPU used during the development phase. Consequently, our MonteCarlo program is only optimized on an NVIDIA Quadro K620 card. To compare our performance with other GPU devices, a specific Monte-Carlo program should be developed and optimized for each individual device.

Although it remains difficult to compare this value to the accelerations obtained in other studies [17, 42-53] due to different computing capacities and simulations, the acceleration provided by the Monte-Carlo method presented in this study is sufficient to enable use of the algorithm in routine clinical settings.

Finally, the Monte-Carlo model presented here was validated using a device dedicated to intraoperative PDT, specifically a $70 \mathrm{~mm}$ long cylindrical diffuser located at the center of a balloon. The closeness of the results to the experimental data reinforce the validity of the Monte-Carlo algorithm.

PDT dosimetry is a multifactor estimation used to compute impact on tissues. Predictive dosimetry includes computing the light, PS and oxygen distribution in biological tissues. Although it is highly time-consuming, the Monte-Carlo method remains the standard method for computing the propagation of light in heterogeneous media. Monitoring PDT treatment includes several features (PS fluorescence, detection of singlet oxygen) to improve the "PDT 
dose" metric. Finally, a collaborative effort between biologists, chemists, physicists, and engineers will lead to a comprehensive definition of the PDT dose [58].

Author contribution: Clément DUPONT designed the study, performed the analyses and wrote the paper. Grégory BAERT contributed to the creation of the Monte-Carlo code and its optimization. Serge MORDON and Maximilien VERMANDEL supervised the study and reviewed the paper.

Conflict of Interest Statement: none 
1. Pahernik, S.A., et al., Distribution and pharmacokinetics of Photofrin ${ }^{\circledR}$ in human bile duct cancer. Journal of Photochemistry and Photobiology B: Biology, 1998. 47(1): p. 58-62.

2. Orenstein, A., et al., A comparative study of tissue distribution and photodynamic therapy selectivity of chlorin e6, Photofrin II and ALA-induced protoporphyrin IX in a colon carcinoma model.British Journal of Cancer, 1996. 73(8): p. 937-944.

3. Marchal, S., Stratégies pro-apoptotiques appliquées au traitement photodynamique avec le Foscan ${ }^{\circledR}$ de modèles précliniques d'adénocarcinome humain. 2008, Université Henri Poincaré - Nancy I.

4. Kostron, H., T. Fiegele, and E. Akatuna, Combination of FOSCAN ${ }^{\circledR}$ mediated fluorescence guided resection and photodynamic treatment as new therapeutic concept for malignant brain tumors. Medical Laser Application, 2006. 21(4): p. 285-290.

5. Tetard, M.C., et al., Experimental use of Photodynamic Therapy in high grade gliomas: a review focused on 5-aminolevulinic acid. Photodiagnosis Photodyn Ther, 2014. 11(3): p. 19.

6. Angell-Petersen, E., et al., Influence of light fluence rate on the effects of photodynamic therapy in an orthotopic rat glioma model. J Neurosurg, 2006. 104(1): p. 109-17.

7. Baran, T.M. and T.H. Foster, Comparison of flat cleaved and cylindrical diffusing fibers as treatment sources for interstitial photodynamic therapy. Med Phys, 2014. 41(2): p. 022701.

8. Sandell, J., et al., A Treatment Planning System for Pleural PDT. Proceedings of SPIE--the International Society for Optical Engineering, 2010. 7551: p. 75510C.

9. Cassidy, J., V. Betz, and L. Lilge, Treatment plan evaluation for interstitial photodynamic therapy in a mouse model by Monte Carlo simulation with FullMonte. Frontiers in Physics, 2015. 3: p. 1-10.

10. Cassidy, J., V. Betz, and L. Lilge. Monte Carlo fluence simulation for prospective evaluation of interstitial photodynamic therapy treatment plans. in Proc. SPIE Optical Methods for Tumor Treatment and Detection: Mechanisms and Techniques in Photodynamic Therapy XXIV. 2015.

11. Betrouni, N., et al., An image guided treatment platform for prostate cancer photodynamic therapy. Conf Proc IEEE Eng Med Biol Soc, 2013. 2013: p. 370-3.

12. Shafirstein, G., et al., Interstitial Photodynamic Therapy-A Focused Review. Cancers (Basel), 2017. 9(2): p. 14.

13. Jacques, S.L. and B.W. Pogue, Tutorial on diffuse light transport. Journal of Biomedical Optics, 2008. 13(4): p. 041302.

14. Zhu, C. and Q. Liu, Review of Monte Carlo modeling of light transport in tissues. Journal of Biomedical Optics, 2013. 18(5): p. 13.

15. Fang, Q. and D.A. Boas, Monte Carlo simulation of photon migration in $3 D$ turbid media accelerated by graphics processing units. Optics Express, 2009. 17(22): p. 20178-90.

16. Alerstam, E., et al., Next-generation acceleration and code optimization for light transport in turbid media using GPUs. Biomedical Optics Express, 2010. 1(2): p. 658-675.

17. Alerstam, E., T. Svensson, and S. Andersson-Engels, Parallel computing with graphics processing units for high-speed Monte Carlo simulation of photon migration. Journal of Biomedical Optics, 2008. 13(6): p. 060504-060504-3.

18. Dupont, C., et al., 5-ALA Photodynamic Therapy in Neurosurgery, Towards the Design of a Treatment Planning System: A Proof of Concept. IRBM, 2017. 38(1): p. 34-41.

19. Porz, N., et al., Multi-modal glioblastoma segmentation: man versus machine. PLoS One, 2014. 9(5): p. e96873.

20. Jacques, S., T. Li, and S. Prahl. mcxyz. 2013 [cited 2017; Available from: http://omlc.org/software/mc/mcxyz/index.html.

21. Jacques, S.L., Coupling 3D Monte Carlo light transport in optically heterogeneous tissues to photoacoustic signal generation. Photoacoustics, 2014. 2(4): p. 137-142.

22. Cassidy, J., et al., High-performance, robustly verified Monte Carlo simulation with FullMonte. J Biomed Opt, 2018. 23(8): p. 1-11. 
23. Rendon, A., J.C. Beck, and L. Lilge, Treatment planning using tailored and standard cylindrical light diffusers for photodynamic therapy of the prostate. Phys Med Biol, 2008. 53(4): p. 113149.

24. Murrer, L.H., J.P. Marijnissen, and W.M. Star, Ex vivo light dosimetry and Monte Carlo simulations for endobronchial photodynamic therapy. Phys Med Biol, 1995. 40(11): p. 180717.

25. Farina, B., et al., Monte Carlo simulation of light fluence in tissue in a cylindrical diffusing fibre geometry. Phys Med Biol, 1999. 44(1): p. 1-11.

26. Beck, T.J., et al., Interstitial photodynamic therapy of nonresectable malignant glioma recurrences using 5-aminolevulinic acid induced protoporphyrin $I X$. Lasers in Surgery and Medicine, 2007. 39(5): p. 386-93.

27. Baran, T.M. and T.H. Foster, New Monte Carlo model of cylindrical diffusing fibers illustrates axially heterogeneous fluorescence detection: simulation and experimental validation. Journal of Biomedical Optics, 2011. 16(8): p. 085003-085003-11.

28. Murrer, L.H., H.P. Marijnissen, and W.M. Star, Monte Carlo simulations for EndoBronchial Photodynamic Therapy: the influence of variations in optical and geometrical properties and of realistic and eccentric light sources. Lasers Surg Med, 1998. 22(4): p. 193-206.

29. Amdahl, G.M., Validity of the Single Processor Approach to Achieving Large Scale Computing Capabilities (Reprinted from the AFIPS Conference Proceedings, 1967). IEEE Solid-State Circuits Society Newsletter, 2007. 12(3): p. 19-20.

30. Hill, M.D. and M.R. Marty, Amdahl's Law in the Multicore Era. Computer, 2008. 41(7): p. 3338.

31. Ninni, P.D., F. Martelli, and G. Zaccanti, Intralipid: towards a diffusive reference standard for optical tissue phantoms. Phys Med Biol, 2011. 56(2): p. N21-8.

32. Aernouts, B., et al., Dependent scattering in Intralipid ${ }^{\circledast}$ phantoms in the 600-1850 nm range. Optics Express, 2014. 22(5): p. 6086-6098.

33. Stringasci, M.D., et al., Interstitial PDT using diffuser fiber-investigation in phantom and in vivo models. Lasers Med Sci, 2017.

34. Spinelli, L., et al., Determination of reference values for optical properties of liquid phantoms based on Intralipid and India ink. Biomedical Optics Express, 2014. 5(7): p. 2037-2053.

35. Georges, W., et al., An optical phantom with tissue-like properties in the visible for use in PDT and fluorescence spectroscopy. Physics in Medicine and Biology, 1997. 42(7): p. 1415.

36. Stepp, H., et al., ALA and Malignant Glioma: Fluorescence-Guided Resection and Photodynamic Treatment. Journal of Environmental Pathology, Toxicology and Oncology, 2007. 26(2): p. 157-164.

37. Johansson, A., et al., Interstitial Photodynamic Therapy of Brain Tumors. IEEE Journal of Selected Topics in Quantum Electronics, 2010. 16(4): p. 841-853.

38. Rühm, A., et al. 5-ALA based photodynamic management of glioblastoma. in Proc. of SPIE Optical Techniques in Neurosurgery, Neurophotonics, and Optogenetics. 2014.

39. Marijnissen, J.P. and W.M. Star, Calibration of isotropic light dosimetry probes based on scattering bulbs in clear media. Phys Med Biol, 1996. 41(7): p. 1191-208.

40. Marijnissen, J.P. and W.M. Star, Performance of isotropic light dosimetry probes based on scattering bulbs in turbid media. Phys Med Biol, 2002. 47(12): p. 2049-58.

41. Zhu, T. AAPM recommendations for in-vivo light dosimetry for Photodynamic Therapy. in 16th International Photodynamic Association World Congress 2017. Comibra, Portugal.

42. Hetzel, F.W., et al., AAPM REPORT NO. 88: PHOTODYNAMIC THERAPY DOSIMETRY. 2005, American Association of Physicists in Medicine.

43. Dupont, C., et al., A novel device for intraoperative photodynamic therapy dedicated to glioblastoma treatment. Future Oncology, 2017.

44. Dupont, C., Photodynamic therapies of high-grade gliomas: from theory to clinical perspectives. 2017, Université du Droit et de la Santé - Lille II. p. 238. 
45. Dupont, C., et al., INtraoperative photoDYnamic Therapy for GliOblastomas: Study Protocol for a Phase I Clinical Trial. Neurosurgery, 2018: p. nyy324-nyy324.

46. Cheong, W.F., S.A. Prahl, and A.J. Welch, A review of the optical properties of biological tissues. IEEE Journal of Quantum Electronics, 1990. 26(12): p. 2166-2185.

47. Vo-Dinh, T., Biomedical photonics handbook. 2003, Boca Raton, Fla.: CRC Press. 1787.

48. Martinsen, P., et al., Accelerating Monte Carlo simulations with an NVIDIA ${ }^{\circledR}$ graphics processor. Computer Physics Communications, 2009. 180(10): p. 1983-1989.

49. Carbone, N., et al., Solution of the direct problem in turbid media with inclusions using Monte Carlo simulations implemented in graphics processing units: new criterion for processing transmittance data. Journal of Biomedical Optics, 2010. 15(3): p. 035002-035002-9.

50. Leung, T.S. and S. Powell, Fast Monte Carlo simulations of ultrasound-modulated light using a graphics processing unit. Journal of Biomedical Optics, 2010. 15(5): p. 055007-055007-7.

51. Ren, N., et al., GPU-based Monte Carlo simulation for light propagation in complex heterogeneous tissues. Optics Express, 2010. 18(7): p. 6811-23.

52. Cai, F. and S. He, Using graphics processing units to accelerate perturbation Monte Carlo simulation in a turbid medium. Journal of Biomedical Optics, 2012. 17(4): p. 04050210405023.

53. Powell, S. and T.S. Leung, Highly parallel Monte-Carlo simulations of the acousto-optic effect in heterogeneous turbid media. J Biomed Opt, 2012. 17(4): p. 045002.

54. Glaser, A.K., et al., A GAMOS plug-in for GEANT4 based Monte Carlo simulation of radiationinduced light transport in biological media. Biomed Opt Express, 2013. 4(5): p. 741-59.

55. Yang, O. and B. Choi, Accelerated rescaling of single Monte Carlo simulation runs with the Graphics Processing Unit (GPU). Biomed Opt Express, 2013. 4(11): p. 2667-72.

56. Gorshkov, A.V. and M.Y. Kirillin, Acceleration of Monte Carlo simulation of photon migration in complex heterogeneous media using Intel many-integrated core architecture. Journal of Biomedical Optics, 2015. 20(8): p. 085002-085002.

57. Li, P., et al., GPU acceleration of Monte Carlo simulations for polarized photon scattering in anisotropic turbid media. Applied Optics, 2016. 55(27): p. 7468-7476.

58. Zhu, T.C. and J.C. Finlay, The role of photodynamic therapy (PDT) physics. Medical Physics, 2008. 35(7): p. 3127-36. 